ORIGINAL

\title{
Síndrome de Ekbom en consumidor de metilfenidato intravenoso
}

\section{Ekbom Syndrome in an intravenous methylphenidate abuser}

César Pereiro Gómez ; Javier Vicente-Alba*;

Alberto Ramos-Caneda ${ }^{* *}$; Carlos Vázquez Ventoso ${ }^{* *}$;

Eva Fontela-Vivanco*; juan Carlos Díaz del Valle ${ }^{* *}$
* Unidad Asistencial de Drogodependencias de A Coruña.

${ }^{\star *}$ Complejo Hospitalario Universitario de A Coruña.
Enviar correspondencia a:

César Pereiro Gómez. Unidad Asistencial de Drogodependencias de A Coruña.

Calle Ermita 2a (Polígono de A Grela). 15008 A Coruña

cesarpereiro@telefonica.net

\section{Resumen}

El Sindrome de Ekbom es un trastorno mental en que el paciente tiene la convicción delirante monotemática de estar infectado por parásitos. Se trata de un cuadro poco frecuente, que inicialmente fue estudiado por dermatólogos. No se conoce exactamente la etiopatiogenia hasta el momento, si bien se han objetivado diversas causas: enfermedades metabólicas (entre otras causas físicas), cuadros psiquiátricos, fármacos, etc. En la actualidad, se ha encontrado relación entre el consumo de tóxicos y cuadros psicóticos, que parecen debidos a la alteración de la Dopamina a nivel de sus receptores. En este artículo realizamos una revisión del cuadro clínico y presentamos el caso clínico de un paciente consumidor de metilfenidato intravenoso que desarrolla un delirio de infestación.

Palabras clave: ekbom, parasitosis, infestación, delirio, metilfenidato.

\section{Abstract}

Ekbom syndrome is a mental disorder in which the patient has the monothematic delusion of being infected by parasites. It is an uncommon condition that was initially studied by dermatologists. The exactly etiology is unknown to date, though several causes have been proposed, including metabolic diseases (among other physical causes), psychiatric disorders, drugs, etc. Research has now found a relationship between drug abuse and psychotic symptoms, which appear to be due to altered levels of dopamine at the receptor level. In this article we review the clinical features of the condition and present the case report of an intravenous methylphenidate abuser who developed a delusion of parasitosis.

Key words: Ekbom, parasitosis, infestation, delusion, methylphenidate. 
$\mathrm{E}$ Síndrome de Ekbom es un trastorno mental en que el paciente tiene la convicción delirante monotemática de estar infectado por parásitos (Huergo y Fernández, 2007). No debe ser confundido con el Síndrome de Wittmaack-Ekbom, también denominado Síndrome de piernas inquietas (Hinkle, 2010).

Fue descrito en 1938 por el autor que da nombre al cuadro tras la observación de 7 casos en mujeres posmenopáusicas sin antecedentes psiquiátricos relevantes. Así, Ekbom fue el primero en describir las causas, el comportamiento y pronóstico, usando el término "deliro presenil parasitario dermatozoico" (Moreno, Ponce, Narváez y Ubbelohde, 2007). Sin embargo, es probable que fuera ya descrito en 1894 en Francia por Thiedierge bajo el nombre de "acarophobie" y en la literatura médica se observa la descripción de cuadros similares bajo distintas denominaciones tales como parasitosis, delirio de infestación, delusio parasitorum, delirio parasitario dermatozoico, síndrome de la caja de cerillas, etc. (Moreno et al., 2007). Aunque Thibierge no fue el único en describirlo como fobia -al cuadro también se le ha denominado dermatofobia, parasitofobia, acarofobia-, no parece este término el más adecuado ya que no solemos encontrar conductas evitativas de la fobia como tal.

En la actualidad existen ciertas discrepancias en cuanto dónde situar este sindrome en el sistema clasificatorio (Rodríguez-Cerdeira, Telmo y Arenas, 2010), planteándose incluso la validez como entidad nosológica o como un cuadro que aparece superpuesto a otras patologías psiquiátricas (Musalek, Bach M, Passweg y Jaeger, 1990). En la actualidad se clasifica como un Trastorno de ideas delirantes persistentes de tipo somático F.22.0 en el CIE-10 (WHO, 2007) y como Trastorno delirante de tipo somáticio F22.00 (APA, 1994).

Las publicaciones de series de casos son escasas (tabla 1), y suelen ser presentadas por dermatólogos (Hinkle, 2010), que son los médicos a los que suelen recurrir estos pacientes de forma más habitual.

La prevalencia anual se estima en 80 casos por millón de habitantes, con una incidencia de 20 casos por millón

Tabla 1. Estudios de series de casos de delirios de parasitación

\begin{tabular}{cc}
\hline \multicolumn{2}{c}{ Estudios dermatológicos } \\
\hline Wilson y cols (1946, 1952) & 85 casos \\
Schrut y Waldron (1963) & 100 casos \\
Tullet (1965) & 27 casos \\
Aw DCW, Thong Jy (1994) & 8 casos \\
\hline \multicolumn{2}{c}{ Estudios psiquiátricos } \\
\hline Ekbom (1938) & 7 casos \\
Skott (1978) & 57 casos \\
Mester (1982) & 41 casos \\
Valbuena y cols (1984) & 4 casos \\
Munro (1988) & 50 casos \\
Bathia MS (2000) & 55 casos \\
Wenning MT, Davy LE (2003) & 5 casos
\end{tabular}

(Zamora et al., 2011). No existen datos sociodemográficos representativos en estos pacientes y algunos autores estiman que la incidencia puede ser mucho mayor a la descrita (Hinkle, 2010). La mayoría son personas mayores y mujeres, aunque también se ha descrito en jóvenes. En mayores de 50 años la distribución por sexos es 2:1 para las mujeres, por debajo de los 50 años la distribución tiende a igualarse. La edad media se ha situado en torno a los 70 años. Esto puede tener explicación en la pluripatología ligada a la edad (diabetes mellitus, hipertensión arterial, disminución de la agudeza visual y acústica...) cuyas manifestaciones, a nivel dermatológico, pueden inducir una interpretación delirante. También se ha visto que existe una relación en este rango de edad con la polifarmacia (Doucet et al., 1996). Esquizofrenia Paranoide, abuso de tóxicos y delirios compartidos, suelen existir en pacientes jóvenes con este síndrome. Se han documentado casos, no obstante, de adolescentes con un delirio de parasitación primario (Leung, Leung y Ungvari. (2004).

La etiopatiogenia del Síndrome de Ekbom es desconocida. Investigaciones recientes han propuesto que lesiones a nivel estriatal y putamen, con inhibición de la función del Transportador Estriatal de Dopamina (DAT), darian lugar a un aumento en los niveles de dopamina extracelular, siendo este aumento el responsable del cuadro clínico (Huber, Kirchler, Karner y Pycha, 2006; Huber, Karner, Kirchler, Lepping y Freudenmann, 2008).

Tabla 2. Etiología del Síndrome de Ekbom secundario

\begin{tabular}{|c|c|}
\hline \multicolumn{2}{|c|}{ Causas neurológicas } \\
\hline Enfermedad de Alzheimer & Atrofia cortical y subcortical \\
\hline Enfermedad de Huntington & Traumatisco craneal \\
\hline Neurosifilis & Tumores cerebrales \\
\hline Infarto cerebral & Arteriosclerosis cerebral \\
\hline \multicolumn{2}{|c|}{ Causas médicas } \\
\hline Delirium & Hipertensión \\
\hline Diabetes mellitus & Insuficiencia renal \\
\hline Insuficiencia cardiaca & Tuberculosis pulmonar \\
\hline Déficit de Vitamina B12 & Hipoparatiroidismo \\
\hline Linfoma mediastínico & Neoplasias \\
\hline Esclerosis múltiple & Pelagra \\
\hline Desnutrición & Vitiligo \\
\hline Insuficiencia hepática & Hipotiroidismo \\
\hline Deprivación sensorial & Encefalopatia por tóxicos. \\
\hline \multicolumn{2}{|c|}{ Causa exógenas } \\
\hline Cocaina & Corticoides \\
\hline Anfetaminas & Fenelcina \\
\hline Ciprofloxacino & Amantadina \\
\hline Bleomicina & Metilfenidato \\
\hline \multicolumn{2}{|c|}{ Causas psiquiátricas } \\
\hline Psicosis esquizofrénicas & Depresión endógena \\
\hline Estados paranoides & Psicosis somática \\
\hline Retraso mental & Folie à deux \\
\hline Alucinosis alcohólica crónica & Delirium tremens \\
\hline Psicosis hipocondriaca monosintom & \\
\hline
\end{tabular}


Podemos clasificar los delirios de parasitación en Primarios o Secundarios, según su origen. Los delirios de parasitación primarios, son aquellos en los que no se encuentra una causa física o psíquica que los produzca (es por tanto un diagnóstico de exclusión). Los secundarios, aparecen en el contexto de otra patología. Es importante descartar que, en efecto, pueda existir una infestación por un parásito real (Hinkle, 2010).

Entre las causas implicadas en la aparición del Ekbom se han descrito factores de tipo endocrinológico (de ahí su posible relación con la menopausia), cardiovascular, hematológico, lesiones del SNC y también de tipo psiquiátrico (retraso mental, trastornos afectivos, esquizofrenia, etc.) (Huergo y Fernández, 2007). También se ha atribuido el origen a causas exógenas, como determinados fármacos (corticoides, antibióticos, etc.) o al consumo de tóxicos (cocaína o anfetaminas) en el contexto una psicosis inducida (Serrano, Miguel, Diaz del Valle y Vázquez, 2001) (tabla 2).

A grandes rasgos el cuadro clínico que configura el Síndrome de Ekbom es un delirio de infestación, esto es, la convicción de estar invadido por parásitos. Los pacientes creen que existen organismos que les recorren el cuerpo. Van a estar presentes alucinaciones táctiles (pinchazos, sensación de quemazón, picores, etc.) y visuales, así como recuerdos e interpretaciones delirantes. El prurito es el sintoma más frecuente, que se encuentra en casi el $80 \%$ de los pacientes (Hinkle, 2010).

Los pacientes suelen contar que los parásitos vivían previamente en objetos inanimados (muebles, vehículos, etc.) y de ahí pasan a seres vivos. Saben cuál fue el punto de infestación y es frecuente que este hecho lo asocien a un evento vital importante (el fallecimiento de un familiar, un divorcio, etc.). Pueden atribuir, además, una explicación al hecho de la infestación (que va desde la ingesta de un alimento en mal estado, hasta una idea delirante de tipo paranoide por una confabulación de familiares suyos para hacerles daño, etc) (Hinkle, 2010). Es habitual que describan al parásito, aunque sea invisible, comentan que puede cambiar de color o que tiene una serie de características especiales y que no se ven en insectos "normales" (como que hablen, por ejemplo). Suelen relatar una mayor actividad nocturna, lo que se atribuye a problemas de insomnio (Hinkle, 2011). Es frecuente que sepan su ciclo vital y cómo cambian a medida que evolucionan. Acostumbran a mostrar las lesiones que tienen en el cuerpo como pruebas fehacientes de la existencia de los parásitos y emplean gran parte del tiempo en intentar capturar uno o recoger muestras, que van a guardar en cajas de cerillas (de ahí el nombre del síndrome), bolsas o en botes. (Hinkle, 2010). La topografía de las lesiones corresponde a sitios que están al alcance del paciente. Suelen ser asimétricas, con predomino de acuerdo con la mano dominante; morfología variable, pueden mostrar huella de rascado, escoriaciones, liquenificación, úlceras, fisuras, datos de dermatitis de contacto y cicatrices (Moreno et al., 2007). Son producidas por los pacientes al intentar extraer los parásitos. Es frecuente que esto se produzca utilizando las uñas o los dientes, pero pueden hacerlo con cuchillos, cuchillas de afeitar y cualquier otro objeto afilado (Koo y Lee, 2001), Ilegando a producirse lesiones severas, echarse petróleo por encima, ingesta de pesticidas, disolvente, etc. (como el caso clínico que presentamos). La limpieza de la casa y de la ropa de manera obsesiva es frecuente (Le, 2003). Pueden cambiar de domicilio y de trabajo en varias ocasiones, e incluso llegar a quemar o inundar su domicilio, buscando destruir el parásito. (Moreno et al., 2007). La duración del cuadro, en general, es de meses a años. En un estudio se vio que el $20 \%$ de una serie de pacientes llevaba 5 años de evolución (Reilly y Batcherlor, 1986) .

Hay que destacar la repercusión afectiva de estos pacientes, que se sienten "desesperados" (Donabedian, 2007) ante su situación, acudiendo en múltiples ocasiones a distintos especialistas sin éxito. Pueden llegar a expresar ideas suicidas (Hillert, Gieler, Niemeier y Brosing, 2004), llegando a realizar tentativas suicidas (Aw, Thong y Chan, 2004). Lyel, en su estudio (Lyel, 1983), cita 5 casos de pacientes con este Sindrome que se suicidaron, poniendo énfasis en la patología psiquiátrica que presentaban como factor principal. No es extraño (ocurre entre un 5 y un 15\% de las ocasiones) que el caso se presente como un delirio compartido o folie a deux en las personas cercanas al paciente (Moreno et al., 2007).

El abordaje debe ser un abordaje multidisciplinar y es complicado en ocasiones. El mayor problema es establecer una buena alianza terapéutica con pacientes (Hinkle, 2010), que no tienen ninguna conciencia, por definición, de la enfermedad. La idea de que nadie les cree y la sensación de ser unos incomprendidos puede hacer que sus ideas paranoides empeoren (Wenning, Davy, Catalano y Catalano, 2003). Se recomienda una intervención temprana, antes de que el cuadro delirante progrese (Hinkle, 2010). Los antipsicóticos son el tratamiento de primera elección (Alonzo, Fernández, Campos y Espinosa, 2008). Tradicionalmente se ha recomendado Pimocide, que actúa bloqueando los receptores dopaminérgicos D2 (Stahl, 2009), aunque estudios actuales muestran resultados similares con antipsicóticos de segunda generación: Olanzapina (Freudenmann, Schonfeldt y Lepping, 2007; Meehan, 2006), Risperidona (Pacan, Reich y Szepietowski, 2004), Aripiprazol (Rocha y Hara, 2007) y Amisulprida (Lepping, Gil-Candon y Freudenmann, 2005). Estos últimos, contribuyen a mantener la adherencia terapéutica por su buena tolerabilidad y bajo perfil de efectos secundarios (Poli y Agrimi, 2009). Dada la posible larga duración de los síntomas, se recomienda una dosis mínima de mantenimiento (Hinkle, 2010). Es importante explorar la posibilidad que exista patología psiquiátrica comórbida, como es el caso de trastornos afectivos o trastornos de ansiedad. (Alonzo, Fernández, Campos y Espinosa, 2008).

\section{Descripción del Caso Clínico}

Presentamos el caso clínico de un paciente a tratamiento en la Unidad Asistencial de Drogodependencias de A Coruña, de 45 años, soltero, con pareja estable y que vive solo. Le lleva al Servicio de Urgencias un familiar por un cuadro de ansiedad reactiva a una idea de infestación. Al realizar la historia clínica, en sus antecedentes persona- 
les se registran los siguientes datos: Infección por VIH (A2) a tratamiento con TARGA y Candidiasis oral. Último control analítico (Octubre 2010): CD4 218, ARN-VIH: $21480 \mathrm{cp} / \mathrm{ml}$. Hepatitis crónica por VHC y VHB. Trombopenia autoinmune severa, probablemente secundaria a infección por VIH. Bronquiectasias. Ingresado en 2005 por respectivos episodios de celulitis en MID secundaria a venopunción y gastroenteritis aguda enteroinvasiva. Ingresado en 2010 por Fiebre que no llegó a filiarse por alta voluntaria. En cuanto a su historia adictiva, está tratamiento con metadona desde hace varios años (actualmente toma $35 \mathrm{mg} / \mathrm{día}$ ) y clonacepam (máximo $6 \mathrm{mg} / \mathrm{dia}$ ) y según relata, en los últimos meses ha comenzado a consumir de manera continuada metilfenidato por vía intravenosa, llegando a tomar una caja de comprimidos al día. Niega consumo de otros tóxicos.

En lo que atañe al episodio que motiva su visita a urgencias, refiere que desde hace más o menos un mes se encuentra más ansioso y desde hace una semana nota que su casa ha sido invadida por unos insectos. Empezó viéndolos en el suelo, "eran blancos y salían de la madera". La invasión de los insectos se va extendiendo hasta llegar a infestarlo, "se me han metido por las uñas". Comenta que una persona le dijo que son insectos especiales, "atacan al lado del cuerpo que no utilizas. Como soy diestro los tengo sobre todo en el lado izquierdo". Nota como los insectos le recorren el cuerpo. Para sacarlos se produce lesiones cutáneas de distinta gravedad que se localizan en MMSS y MMII (fotos 1 y 2). Ha utilizado gasoil, alcohol, disolvente y otros productos inflamables por toda la casa "para librarme de ellos". Ha llegado a echarse gasolina por el cuerpo para este fin. Trae una caja de cerillas con restos de piel "traigo los bichos, son como ácaros". En su estancia en Urgencias es valorado por Cirugía plástica para valoración de las úlceras realizando curas e indicando tratamiento con amoxicilina y clavulánico.

Refiere consumo de Metilfenidato i.v., unos $30 \mathrm{cps} /$ día de $10 \mathrm{mg}$ desde hace varios meses, aunque "ahora lleva unos días sin tomar nada". Niega consumo de otras sustancias que no sean metadona y clonazepam indicados a dosis terapéuticas en la UAD y los urinoanálisis parecen refrendarlo.

Dado la situación del paciente se decidió ingreso en la Unidad de Hospitalización Psiquiátrica para filiación del cuadro y ajuste del tratamiento psicofarmacológico.

En cuanto a la exploración psicopatológica, estaba consciente, orientado en las tres esferas, colaborador, presentando elevados niveles de ansiedad y angustia. Aspecto desaseado. Caquéctico. Discurso espontáneo, fluido, coherente, centrado en temática delirante que expresaba con elevada resonancia afectiva, sin que existiese crítica, manteniéndose irreductible al razonamiento (ideación delirante de infestación parasitaria). No se objetivan alucinaciones auditivas, sí posibles alucinaciones visuales/interpretaciones delirantes (ve a los insectos). Alucinaciones de tipo cenestésico en los que el paciente nota como los bichos recorren su cuerpo. No se aprecia clínica que indique un trastorno afectivo. No ideación autolítica. Apetito irregular. Insomnio mixto, que progresivamente ha empeorado en esta última semana hasta hacerse global.
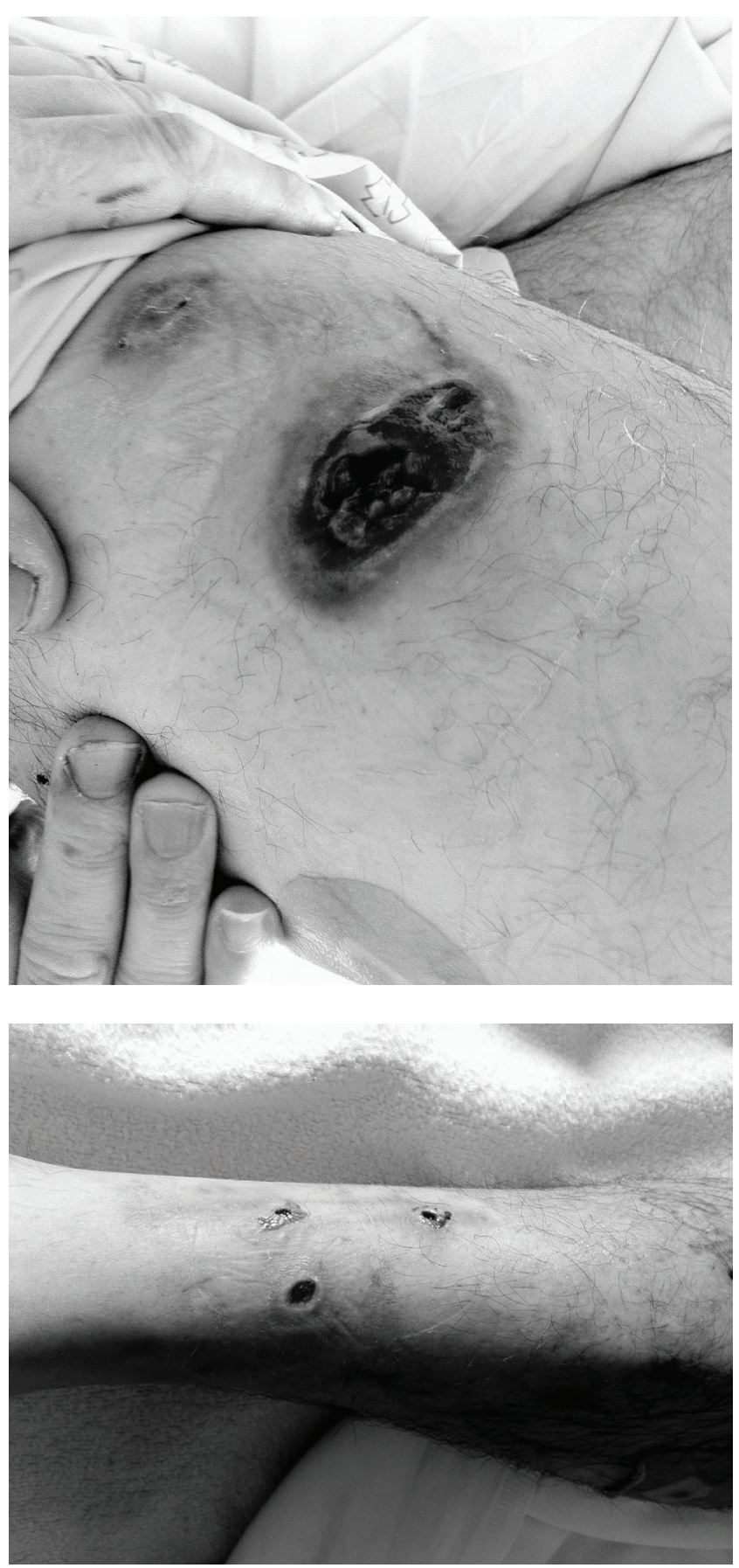

Por lo que atañe a las exploraciones complementarias realizadas, se hizo un TAC craneal que mostró un moderado patrón atrófico difuso sin anomalias agudas. Los análisis toxicológicos, realizados ya en Urgencias, mostraron resultados positivos para metadona y benzodiacepinas, y negativos para otros tóxicos, (incluidos antidepresivos y anfetaminas). Se realizó, durante su estancia, un Test Mini Mental con resultado de 29/30.

Durante el ingreso hospitalario se instauró tratamiento psicofarmacológico con Olanzapina, Amisulpride y Clonazepam a dosis habituales. Al día siguiente del ingreso el paciente presenta fiebre de $38^{\circ}$, que cede con paracetamol, tos con expectoración verdosa y sensación disneica. Se le realiza una radiografía de tórax en el que se objetivam infiltrados pulmonares compatibles con infección aguda. En las analíticas 
seriadas se observan datos de infección y datos de inmunodepresión esperables en paciente seropositivo (Linfocitos CD4 238), con serología de Lúes negativa. Es valorado por los internistas que filian el cuadro como una Neumonía adquirida en la comunidad, tras descartar una posible Tuberculosis pulmonar (tinción de auraminas seriadas en esputo negativas y realización de Mantoux negativo). Se ajustó el tratamiento antibiótico, observándose una buena evolución del cuadro respiratorio con remisión sintomática. La ideación de parasitosis evoluciona de forma favorable, con clara disminución de las repercusiones conductuales y con critica relativa del episodio de parasitación. A las tres semanas del ingreso se decide alta hospitalaria para proseguir seguimiento ambulatorio. Al alta se mantiene tratamiento con Amisulpride a dosis de $800 \mathrm{mg} /$ día, Olanzapina $10 \mathrm{mg} /$ día y Clonazepam $6 \mathrm{mg} / \mathrm{dia}$, manteniendo Metadona y TARGA como previamente realizaba. El diagnóstico realizado es Delirio de Parasitosis-Síndrome de Ekbom en paciente con Dependencia de Opiáceos a tratamiento con sustitutivos, Abuso de Metilfenidato y Benzodiacepinas.

Tras varios meses después del alta, a pesar del tratamiento antipsicótico, se constata que la clínica delirante permanece presente si bien las repercusiones conductuales se han atenuado de forma importante. Así, permanece irreductible la convicción delirante sobre la presencia de la infestación así como de la causa de la misma que se atribuye a una maniobra de sus familiares para dañarle en relación con una herencia en litigio.

\section{Discusión}

El caso que nos ocupa ha sido diagnosticado de Delirio de infestación secundario a consumo de sustancias. No es, sin embargo, una conclusión libre de dudas, pues el diagnóstico diferencial con el Síndrome de Ekbom sigue sin quedar excesivamente claro. Sin embargo, ni el género ni, sobre todo, la edad del paciente, coinciden con los casos más abundantes de esta patología.

Obviamente, lo primero fue considerar la posible presencia de una infestación real que fue descartada tras estudiar las lesiones del paciente y comprobar cómo su origen se debía exclusivamente a las manipulaciones realizadas por él mismo sin que pudiesen encontrarse parásitos de ningún tipo tanto en la superficie como bajo la piel o en las heridas presentes.

Está descrito en el VIH la existencia de trastornos neuropsiquiátricos, que se atribuyen a la propia enfermedad (trastornos cognitivos asociados a la afectación del SNC, como el complejo Demencia-SIDA), Neoplasias o Infecciones oportunistas (Toxoplasmosis, Meningitis Criptocócica), Fármacos empleados para su tratamiento (Esteroides, Interferón, Zidavudina, Efavirenz...) así como otras causas (Enfermedad de Addison) (Polo, 2004). Nuestro paciente estaba en seguimiento por Consultas Externas de Medicina Interna, siendo tratado y controlado de dicha patología, presentando una buena evolución, sin que se objetivase en consultas previas datos de deterioro cognitivo o de síntomas psicóticos reactivos al tratamiento con TARGA.

El estado nutricional basal del paciente (caquéctico), sus condiciones físicas y la presencia de una neumonía nos obligaron de entrada a descartar la posible organicidad como causa de los sintomas psicóticos. Es frecuente que, en el contexto de cuadros confusionales/Delirium, se produzcan síntomas de estirpe psicótica. En relación con esto, el paciente se muestra en todo momento consciente y orientado en las 3 esferas, sin que se objetiven fluctuaciones a nivel de conciencia ni alteraciones mnésicas relevantes. Del mismo modo no pudimos atribuir la sintomatología observada a un problema hepático ya que aún tratándose de un paciente con una hepatopatía crónica, no existía descompensación alguna ni estaba recibiendo tratamiento con interferón u otros fármacos para los que se ha descrito una asociación con cuadros de tipo psicótico (Robaeys, De Bie, Van Ranst y Buntinx, 2008).

Descartamos también un posible delirium relacionado con abstinencia a benzodiacepinas y opiáceos, al no tener constancia del cese del consumo en días anteriores ni tampoco de drásticas reducciones de dosis.

No se objetivaron tanto en Consultas Externas de Dermatología ni de Medicina Interna causa orgánica que pudiese ser responsable de prurito y que el paciente pudiera estar interpretado de manera delirante.

En cuanto al diagnóstico de Psicosis inducida, aunque parece constatable la abstinencia en el consumo de estimulantes los días previos al ingreso, no debemos pasar por alto que es precisamente en este hecho donde nos encontramos la mayor disyuntiva. Mientras el DSM-IV-TR descartaría el diagnóstico, al aparecer ésta tras una interrupción del consumo mayor a un mes, y persistiendo tras el tratamiento, cabe recordar que la CIE-10 alarga el periodo hasta los 6 meses. Además, sólo se puede asegurar con rotundidad una abstinencia previa de otros tóxicos que el paciente había consumido, dada la imposibilidad de practicar analíticas que detecten el consumo de metilfenidato en nuestro medio ambulatorio.

Es bien conocido que la sintomatología presentada por nuestro paciente, donde destaca el delirio de infestación y formicación, son muy característicos del abuso de psicoestimulantes. Este hecho redunda en nuestra decisión de presentar al consumo continuado de metilfenidato como responsable del cuadro. Dado que en periodos de consumo previos de dicha sustancia el paciente no había presentado sintomatología psicótica aparente, podríamos hablar de un trastorno psicótico por sensibilización, característico también de dichos tóxicos. Conviene recordar que el cuadro aparece tras varios meses de consumo y no en sus primeras ocasiones.

En cuanto a la participación de los tóxicos en el origen del cuadro, está bien estudiada la relación entre el consumo habitual de cocaína y múltiples lesiones de indole dermatológica como las vasculitis necrotizantes (Brust, 1997; Chevalier, Rostoker y Larget-Piet, 1995), las vasculitis de Schölein- 
Henoch (Lu y High, 2007), pseudovasculitis (Friedman yWolfsthal, 2005) o vasculitis de Churg-Strauss.

Dentro del diagnóstico diferencial no podemos obviar la posibilidad de un trastorno delirante primario. Los datos clínicos que nos hacen apoyar el diagnóstico de Trastorno Psicótico secundario son, entre otros, la no existencia de síntomas psicóticos previos al inicio de consumo, el continuado abuso de psicoestimulantes y la no existencia de antecedentes familiares.

En el análisis que realizamos de la evolución del paciente tras el ingreso, pensamos que la mejoría observada se debió, en alguna medida, al propio entorno de tratamiento en una Unidad Hospitalaria cerrada, en el que probablemente existía un número menor de estímulos activadores. Sin duda el tratamiento con antipsicóticos realizado, aunque no ha eliminado la ideación delirante, si ha contribuido a disminuir los niveles de ansiedad que presentaba el paciente en un primer momento, con claras repercusiones positivas desde el punto de vista de su conducta que se ve menos condicionada por el delirio parasitario.

Desde el punto de vista neurobiológico podemos hipotetizar que el cuadro clínico observado, sobre la base de una posible vulnerabilidad cerebral previa, pudo relacionarse con el consumo de metilfenidato por vía intravenosa, cuyo efecto más inmediato es un incremento de los niveles de dopamina en los espacios intersinápticos neuronales en varias áreas cerebrales. En relación con esto es interesante comentar que recientemente se ha formulado la hipótesis de que el Síndrome de Ekbom sea debido a una disminución de la actividad del Transportador Estriatal de Dopamina (DAT) (Huber et al., 2008), que conllevaría una acumulación de dopamina en los cuerpos estriados como etiología del cuadro clínico. Este descenso de la densidad y funcionamiento del DAT sería común al proceso de envejecimiento, propio del Síndrome de Ekbom, y a otras causas.

Los tóxicos, como la cocaína o, en el caso de nuestro paciente, el metilfenidato, actuarían a nivel del núcleo estriado bloqueando la acción del DAT, provocando, por consiguiente, un aumento de los niveles de dopamina en esa localización. Existen variantes del Síndrome de Ekbom secundarios a enfermedades médicas como la enfermedad de Parkinson, enfermedades cerebrovasculares o infecciosas, muestran un descenso de la actividad del ligando del DAT a nivel estriatal.

Para finalizar, señalamos que se ha documentado fielmente el papel jugado por los ganglios basales en las alucinaciones visuales y táctiles, por el aumento de esa dopamina a nivel estriatal (Sil'kis, 2005). Igualmente, los mejores resultados en cuanto al tratamiento de estos pacientes han sido aportados por los antipsicóticos con mayor potencia antidopaminérgica, ya sean de primera generación, como la pimozida o el haloperidol, o también los de segunda generación, como risperidona, olanzapina o amisulpride (Lepping, GilCandon y Freudenmann, 2005).

\section{Conflicto de intereses}

Los autores declaran no tener ningún conflicto de interés.

\section{Referencias}

Alonzo, R. P. L., Fernández, B. R., Campos, F. M. M. y Espinosa, A. R. L. (2008). Delirio de parasitosis. Revista Mexicana de Dermatología, 52, 263-267.

American Psychiatric Association (1994). DSM-IV-TR Diagnostic and statistical manual of mental disorders. Washington: American Psychiatric publishing.

Aw, D. C. W., Thong, J. Y. y Chan, H. L. (2004). Delusional parasitosis: case series of 8 patients and review of the literature. Annals of Academy of Medicine Singapore, 33, 89-94.

Bhatia, M. S. (2000). Delusional parasitosis: a clinical profile. International Journal of Psychiatry in Medicine, 30, 83-91.

Brust, J. C. (1997). Vasculitis owing to substance abuse. Neurol. Clin., $15,945-957$

Calvo, P. (2010). Delirio de formicación. A propósito de un caso. Psiquiatría Biológica. 17, 138-139

Chevalier, X., Rostoker, G., Larget-Piet, B. y Gherardi, R. (1995). Schönlein-Henoch purpura with necrotizing vasculitis after cocaine snorting. Clinical Nephrology, 43, 348-349.

De Ronchi, D., Faranca, I., Forti, P., Ravaglia, G., Borderi, M., Manfredi, R. y Volterra, V. (2000). Development of acute psychotic and HIV-1 infection. International Journal of Psychiatry in Medicine, 30, 173-183.

Donabedian, H. (2007). Delusions of parasitosis. Clinical Infectious Diseases, 45, 31-34.

Doucet, J., Chassagne, P., Trivalle, C., Landrin, I. y Pauty, M. D., et al. (1996). Drug-drug interactions related to hospital admissions in older adults: a prospective study of 1000 patients. Journal of the American Geriatrics Society, 44, 944-948.

Ekbom, K. A. (1938). Der Praesenile dermatozoenwahn. Acta Psychiatrica et Neurologica, 13, 227-259.

Freudenmann, R. W., Schonfeldt, C. y Lepping, P. (2007). Primary delusional parasitosis treated with olanzapine. International Psychogeriatrics, 3, 1-9.

Friedman, D. R. y Wolfsthal, S. D. (2005). Cocaine-induced pseudovasculitis. Mayo Clinic Proceedings, 80, 671-673.

Hillert, A., Gieler, U., Niemeier, V. y Brosing, B. (2004). Delusional parasitois. Dermatology and Psychosomatics, 5, 33-35.

Hinkle, N. C. (2010). Ekbom Syndrome: The challenge of "invisible bug" infestations. Annual Review of Entomology, 55, 77-94. doi:10.1146/annurev.ento.54.110807.090514.

Hinkle N. C. (2011). Ekbom Syndrome: A delusional condition of "Bugs in the skin". Current Psychiatry Reports ,13, 178-186. doi: 10.1007/s11920-011-0188-0.

Huber M., Karner, M., Kirchler, E., Lepping, P. y Freudenmann, R.W. (2008). Striatal lesions in delusional parasitosis revealed 
by magnetic resonance imagining. Progress in NeuroPsychopharmacology \& Biological Psychiatry, 32, 1967-1971. doi: 10.1016/j.pnpbp.2008.09.014

Huber, M., Kirchler, E., Karner, M. y Pycha, R., (2006). Delusional parasitosis and the dopamine transporter. A new insight of etiology? Medical Hypotheses 68, 1351-1358. doi: 10.1016/j. mehy.2006.07.061.

Huergo, C. y Fernández, G., (2007). Delirio de Ekbom. Psiquiatría Biológica 14, 233-235. Localizador web artículo 213.158.

Koo, J. y Lee, C. S., (2001). Delusions of parasitosis: a dermatologist's guide to diagnosis and treatment. American Journal of Clinical Dermatology, 2, 285-290.

Le, L. y Gonski, P. N. (2003). Delusional parasitosis mimicking cutaneous infestation in elderly patients. Medical Journal of Australia, 179, 209-210.

Lepping, P., Gil-Candon, R. y Freudenmann, R. W. (2005). Delusional parasitosis treated with amisulpride. Progress in Neurology and Psychiatry, 9, 12-26.

Leung, T. Y., Leung, C. M.y Ungvari, G. S. (2004). A Chinese adolescent with delusional infestation. Hong Kong Journal of Psychiatry, 14, 23-25.

Lu, L. K. y High, W. A. (2007). Acute generalized exanthematous pustulosis caused by illicit street drugs? Archives of Dermatology, $143,430-431$

Lyel, A. (1983). Delusions of parasitosis: the Michelson lecture. British Journal of Dermatology, 108, 485-499.

Meehan, W. J., Badreshia, S. y Mackley, C. L. (2006). Successful treatment of delusions of parasitosis with olanzapine. Archives of Dermatology, 142, 352-355.

Mester, H. (1981). Der Ungezieferwahn-ein Beitrag über die Ätiologie und den Aufbau dieser Halluzinose. Fortschr. Neurology and Psychiatry, 49, 136-144.

Moreno, K., Ponce, R. M., Narváez, V. y Ubbelohde, T. (2007). Síndrome de Ekbom. Revista Mexicana de Dermatología, 51, 51-56.

Munro (1988). A monosymptomatic hypochondriacal psychosis. British Journal of Psychiatry, 153, 37-40.

Musalek, M., Bach, M., Passweg, V. y Jaeger, S. (1990). The position of delusional parasitosis in psychiatric nosology and classification. Psychopathology, 23, 115-124.

Orriols, R., Munoz, X., Ferrer, J., Huget, P. y Morell, F. (1996). Cocaineinduced Churg-Strauss vasculitis. European Respiratory Journal, 9, 175-177

Pacan, P., Reich, A. y Szepietowski, J. C. (2004). Delusional parasitosis successfully controlled with risperidone - two case-reports. Dermatology and Psychosomatics, 5, 193-195.

Poli, R. y Agrimi, E. (2009). Ekbom's Syndrome: two case reports treated with olanzapine. Clinical Neuropsychiatry, 6, 124-127.

Polo, R.y Sanz, J., (2004). Recomendaciones del PNS sobre el manejo de los trastornos relacionados con la infección VIH. Recomendaciones clínicas del Ministerio de Sanidad, 23, 90-103.
Reilly, T. M. y Batcherlor, D. H. (1986). The presentation and treatment of delusional parasitosis: a dermatological perspective. International Clinical Psychopharmacology, 1, 340-353.

Robaeys, G., De Bie, J., Van Ranst, M. y Buntinx, F. (2007). An extremely rare case of delusional parasitosis in a chronic hepatitis $C$ patient during pegylated interferon alpha- $2 b$ and ribavirin treatment. World Journal of Gastroenterology, 13, 2379-2380

Rocha, F. L. y Hara, C. (2007). Aripiprazole in delusional parasitosis: case report. Progress in Neuro-Psychopharmacology \& Biological Psychiatry, 31, 784-786.

Rodríguez-Cerdeira, C., Telmo, J. y Arenas, R (2010). El sindrome de Ekbom: un trastorno entre la dermatología y la psiquiatría. Revista Colombiana de Psiquiatría, 39, 440-447.

Schrut, A. H. y Waldron, W. G. (1963). Psychiatric and entomological aspects of delusory parasitosis. Journal of the American Medical Association, 186, 4, 213-214.

Skott, A. (1978). Delusion of infectation dermatozoenwahn, Ekbonis syndrome. Reports from the Psychiatric Research Center 13, Gothenburg.

Serrano, M, Miguel, D., Diaz, J. C., Vazquez, C. y Valle, J. C. (2001). Los delirios de infestación parasitaria. Síndrome de Ekbom. Revisión y presentación de un caso. Cuadernos de Atención Primaria, 8 , 275-285.

Sil'kis, I. G. (2005). A role of the basal ganglia in the occurrence of visual hallucinations (a hypothetical mechanism). Zhurnal vysshei nervnoi deiatelnosti imeni I P Pavlova, 55, 592-607.

Stahl, S. M., (2009). The Prescriber's Guide. Stahl's Essential Psychopharmacology. UK: Cambridge University Press (traducción castellana, Madrid: Aulamédica).

Tullet, G. L. (1965). Delusions of parasitosis. British Journal of Dermatology, 77, 448-455.

Vallbuena, A. y Avila, J.J. (1984). Delirio dermatozoico (parasitario). Medicina Clínica, 83, 249-252

Wenning, M. T., Davy, L. E., Catalano, G. y Catalano, M. C. (2003). Atypical antipsychotics in the treatment of delusional parasitosis. Annals of Clinical Psychiatry. 15, 233-239.

Wilson, J. y Miller, H. (1946). Delusions of parasitosis. Archives of Dermatology and Syphilology, 54, 39-56.

WHO (2007). International Classification of diseases (ICD-10). Ginebra: WHO.

Zamora, F.J., Benitez, C., Calderón, P., Guisado, J.A., Teva, M.I. y Sánchez-Waisen, M.R., (2011). Un caso de syndrome de Ekbom. Psiquiatría Biológica. Doi: 10.1016/j.psiq.2010.12.006. 
